\title{
Exploring Flat Faced Longhorn Beetles (Cerambycidae: Lamiinae) from the Reserve Forests of Dooars, West Bengal, India
}

\author{
Sumana Saha, ${ }^{1}$ Hüseyin Özdikmen, ${ }^{2}$ Manish Kanti Biswas, ${ }^{3}$ and Dinendra Raychaudhuri ${ }^{4}$ \\ ${ }^{1}$ Department of Zoology, Darjeeling Government College, Government of West Bengal, Darjeeling, West Bengal 734101, India \\ ${ }^{2}$ Gazi Üniversitesi, Fen-Edebiyat Fakültesi, Biyoloji Bölümü, 06500 Ankara, Turkey \\ ${ }^{3}$ Department of Zoology, Sreegopal Banerjee College, Mogra, Hooghly, West Bengal 712148, India \\ ${ }^{4}$ Entomology Laboratory, Department of Zoology, University of Calcutta, 35 Ballygunge Circular Road, Kolkata, \\ West Bengal 700019, India
}

Correspondence should be addressed to Dinendra Raychaudhuri; dinendrarccu@gmail.com

Received 25 June 2013; Accepted 7 August 2013

Academic Editors: Y. Fan and P. Simões

Copyright (c) 2013 Sumana Saha et al. This is an open access article distributed under the Creative Commons Attribution License, which permits unrestricted use, distribution, and reproduction in any medium, provided the original work is properly cited.

The present study deals with 29 lamiid species under 21 genera of Dooars, West Bengal, India. These include 4 newly recorded species, namely, Macrochenus isabellinus Aurivillius, Aesopida malasiaca Thomson, Pterolophia (Hylobrotus) lateralis Gahan and Nupserha quadrioculata (Thunberg) from India while 16 others (marked by •) from the state.

\section{Introduction}

Subfamily Lamiinae (Cerambycidae) include members of flat faced longhorn beetles that are both xylophagous and phytophagous [1]. The group appears to be well studied [2]. Globally more than 20,248 lamiid species under 3,052 genera are known [3]. Many of the details for each of the species are also provided $[4,5]$.

Despite being important from the stand point of forestry/ pollination/bioindicator, this group of insects is yet to receive serious attention in India more so in North East India, one of the two hotspots of the country, an area certainly rich in floral diversity. It is therefore necessary to refer the early works done on the Indian lamiids [6-35]. However, none of the workers ever extensively dealt with these insects of "DOOARS."

In the study area, Dooars represents the biogeographic provinces of Central Himalayas (2C) and lower gangetic plains (7B) situated between latitudes $26^{\circ} 16^{\prime \prime}$ and $27^{\circ} 00^{\prime \prime}$ north and longitudes $88^{\circ} 04^{\prime \prime}$ and $89^{\circ} 58^{\prime \prime}$ east. It includes 4 reserve forests, namely, Buxa Tiger Reserve (BTR, $760.87 \mathrm{sq}$. km.), Jaldapara Wildlife Sanctuary (JWLS, 216.51 sq. km.), Gorumara National Park (GNP, 79.99 sq. km.), and Chapramari Wildlife Sanctuary (CWLS, 9.6 sq. km.), lying in the foothills of Himalaya.
We (saving the second author) for nearly two decades are involved in the exploration of the long horn beetles of the area. Present communication is one such outcome on the lamiids dealing with 29 species under 21 genera. Four of the 29 species, namely, Macrochenus isabellinus Aurivillius, Aesopida malasiaca Thomson, Pterolophia (Hylobrotus) lateralis Gahan, and Nupserha quadrioculata (Thunberg), are reported as new from the country while 16 others (marked by •) from the state.

\section{Material and Methods}

Initiated in 1993, extremely intense survey is still on in the aforesaid forests for the cerambycids (flat-faced longhorn beetles). Insect samples are collected by hand picking, sweep net, and bush/foliage/herb beating. Many of the samples are collected by operating UV light trap during 18-22 hrs of the day. Further processing of the insect material is done following the recommended practices [36] and studied under Stereozoom Binocular Microscopes, models Zeiss SV11 and Olympus SZX7, each with necessary photographic attachment. Reported species are in the deposition of Entomology Laboratory, Department of Zoology, University of Calcutta. 


\section{Results}

The 29 recorded species of 21 genera under 11 tribes are presented hereunder.

\subsection{Tribe: Acanthocinini}

- Ostedes (Trichostedes) assamana Breuning, 1961 (Figure 1(a)).

Ostedes (Trichostedes) assamana Breuning, 1961, Zs. d. Arbeit. Ost. Ent. 12(3): 135.

Material Examined. 1 ex., Kumargram, BTR, 26.v.2004, coll. S. Sarkar; 1 ex., Bendaki, JWLS, 820. xi. 2002, coll. S. Bhattacharya.

Distribution. India: Assam, West Bengal; Laos [3, 4, 37].

\subsection{Tribe: Agapanthini}

Eucomatocera vittata White, 1846 (Figure 1(b)).

Eucomatocera vittata White, 1846, Ann. Mag. Nat. Hist., $18: 49$.

Material Examined. 1 ex., Tashigaon, BTR, 26.v.2003, coll. S. Sarkar; 1 ex., Rajabhatkhawa, BTR, 17.ix.2010, coll. S. Sarkar; 1 ex., Siltorsa, JWLS, 17.iii.2002, coll. S. Bhattacharya; 1 ex., Kunjanagar, JWLS, 27.iv.2002, coll. S. Bhattacharya; 1 ex., $50 \mathrm{ft}$, JWLS, 1.v.2002, coll. S. Bhattacharya; 1 ex., Moiradanga, JWLS, 3.vi.2002, coll. S. Bhattacharya; 2 ex., NWC, JWLS, 7.vi.2002, coll. S. Bhattacharya; 1 ex., Mendabari, JWLS, 9.vi.2002, coll. S. Bhattacharya; 1 ex., C.C.Line, JWLS, 9.vi.2002, coll. S. Bhattacharya; 3 ex., C.C.Line, JWLS, 14.x.2002, coll. S. Bhattacharya; 1 ex., Siltorsa, JWLS, 19.x.2002, coll. S. Bhattacharya; 1 ex., Baradabri, JWLS, 30.iv.2003, coll. S. Bhattacharya; 1 ex, Malangi, JWLS, 2.vi.2003, coll. S. Bhattacharya; 1 ex., Lankapara, JWLS, 5.vi.2003, coll. S. Bhattacharya; 1 ex., T.E.C., JWLS, 9.vi.2003, coll. S. Bhattacharya; 1 ex., Mantharam, JWLS, 18.x.2003, coll. S. Bhattacharya; 1 ex., Sisamara, JWLS, 20.vi.2003, coll. S. Bhattacharya; 1 ex., Gorumara, GNP, 3.xi.2003, coll. M. K. Biswas; 1 ex., Bichabhanga, GNP, 1.iv.2003, coll. M. K. Biswas; 1 ex., Chapramari, CWLS, 4.xi.2003, coll. M. K. Biswas.

Distribution. India: West Bengal; Laos, Myanmar, Sri Lanka, Taiwan, Thailand, and Vietnam [4, 37].

- Tetraglenes hirticornis (Fabricius, 1798) (Figure 1(c)). Saperda hirticornis Fabricius, 1798, Ent. Syst. Suppl., 148.

Material Examined. 1 ex., Sankos, BTR, 8.v.1995, coll. D. Raychaudhuri; 1 ex., Bhutanghat, BTR, 25.v.1995, coll. S. Saha; 1 ex., Jayanti, BTR, 24.v.1996, coll. D. Raychaudhuri; 1 ex., Daidaighat, JWLS, 8.iv.2002, coll. S. Bhattacharya; 2 ex., NWC, JWLS, 7.vi.2002, coll. S. Bhattacharya; 1 ex., Mendabari, JWLS, 9.vi.2002, coll. S. Bhattacharya; 1 ex., Daidaighat, JWLS, 2.vi.2003, coll. S. Bhattacharya; 1 ex., Baradabri, JWLS, 6.vi.2003, coll. S. Bhattacharya.

Distribution. India: West Bengal; China, Indonesia, Laos, Myanmar, Nepal, and Vietnam [4, 37-39].

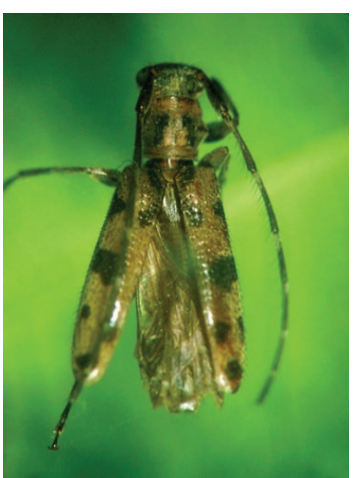

(a)

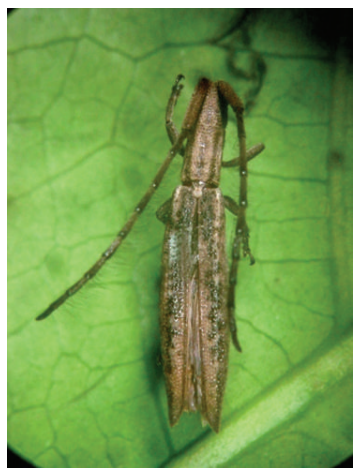

(c)

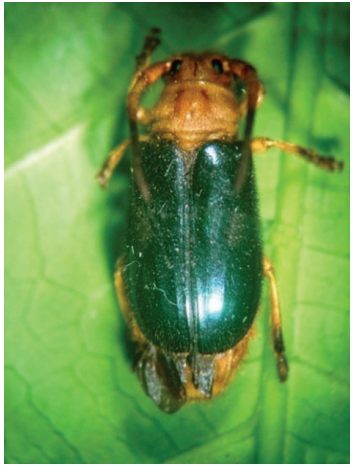

(e)

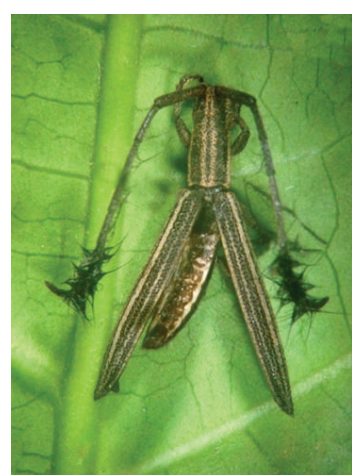

(b)

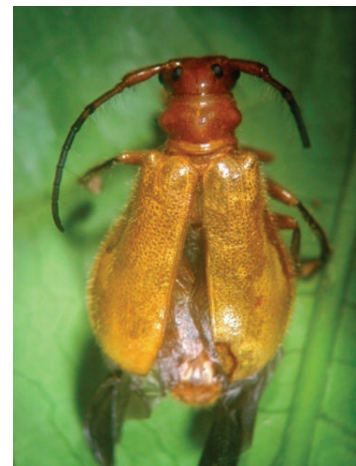

(d)

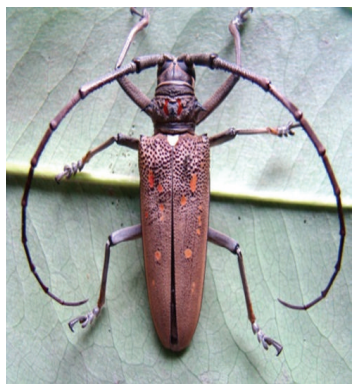

(f)
Figure 1: (a) Ostedes (Trichostedes) assamana Breuning, (b) Eucomatocera vittata White, (c) Tetraglenes hirticornis (Fabricius), (d) Astathes (Tetraophthalmus) gibbicollis Thomson, (e) Astathes (Tetraophthalmus) violaceipennis (Thomson), and (f) Batocera davidis Fairemaire.

\subsection{Tribe: Astathini}

- Astathes (Tetraophthalmus) gibbicollis Thomson, 1865 (Figure 1(d)).

Astathes gibbicollis Thomson, 1865, Syst. Cer., 559.

Material Examined. 1 ex., Poro, BTR, 5.iv.2002, coll. D. Raychaudhuri; 1 ex., Rajabhatkhawa, BTR, 24.v.2009 (LT) coll. S. Sarkar. 


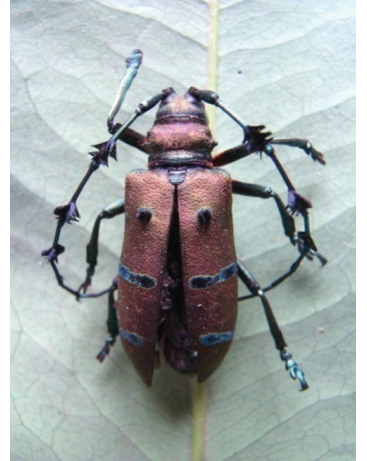

(a)

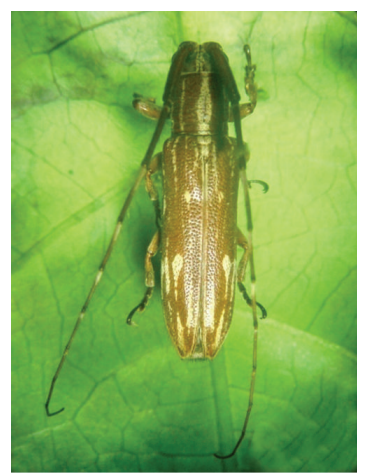

(c)

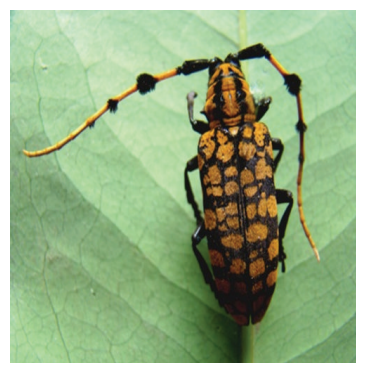

(e)

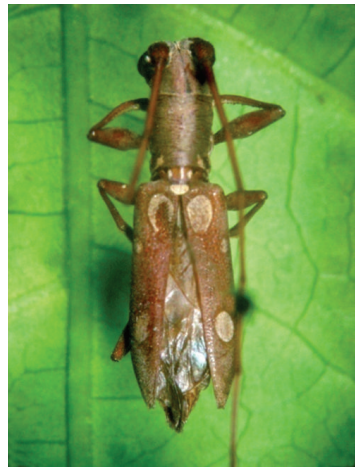

(b)

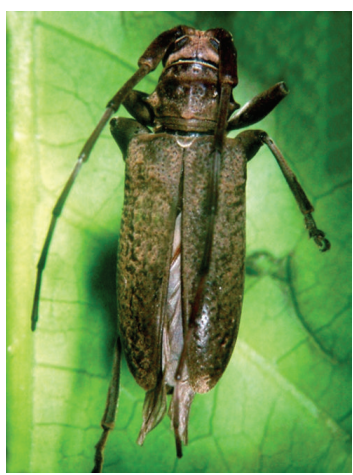

(d)

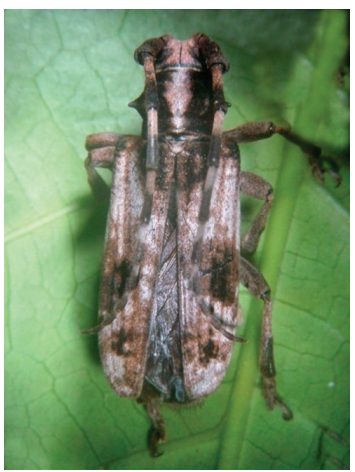

(f)
Figure 2: (a) Diastocera wallichi (Hope), (b) Olenecamptus bilobus (Fabricius), (c) Olenecamptus dominus Thomson, (d) Acalolepta griseipennis (Thomson), (e) Aristobia approximator (Thomson), and (f) Blepephaeus succinator (Chevrolat).

Distribution. India: North India, West Bengal; Laos, Malaysia, Myanmar, and Vietnam [4, 37].

- Astathes (Tetraophthalmus) violaceipennis (Thomson, 1857) (Figure 1(e)).

Tetraophthalmus violaceipennis Thomson, 1857, Archiv. Ent., 1: 53.

Material Examined. 1 ex., Newland, BTR, 26.v.1995, coll. D. Raychaudhuri; 1 ex., Rajabhatkhawa, BTR, 24.v.2009, coll. S. Sarkar; 2 ex., Dima, BTR, 10.iv.2010, coll. S. Sarkar, 1 ex., Dima, BTR, 10.iv.2010, coll. S. Sarkar, 2 ex., Mendabari,
JWLS, 28.iv.2003, coll. S. Bhattacharya; 1 ex., Khunia, GNP, 5.vi.2006, coll. M. K. Biswas.

Distribution. India: Assam, Sikkim, West Bengal; China, Laos, Myanmar, Nepal, Thailand, and Vietnam [4, 38, 40, 41].

\subsection{Tribe: Batocerini}

Batocera davidis Fairemaire, 1878 (Figure 1(f)).

Batocera davidis Fairemaire, 1878, Ann. Soc. Ent. Fr., 8(5): 131.

Materials Examined. 1 ex., Panbari, BTR, 20.v.1995, coll. S. Saha; 1 ex., Jayanti, BTR, 21.iv.2003, coll. S. Sarkar; 1 ex., Buxaduar, BTR, 3.ix.2009(LT), coll. S. Sarkar; 2 ex., Rajabhatkhawa, BTR, 10.ix.2009, coll. S. Sarkar; 1 ex., Gorumara, GNP, 3.11.2003, coll. M. K. Biswas.

Distribution. India: West Bengal; China, Hawaii, Laos, Taiwan, and Vietnam $[4,42]$.

\subsection{Tribe: Ceroplesini}

- Diastocera wallichi (Hope, 1831) (Figure 2(a)).

Lamia wallichi Hope, 1831: Gray's Zool. Misc. 1: 27.

Material Examined. 1 ex., Phaskhawa, BTR, 2.v.1996, coll. S. Saha; 1 ex., Panbari, BTR, 4.iv.2003, coll. S. Bhattacharya.

Distribution. India: Arunachal Pradesh, Assam, Bihar, Meghalaya, Sikkim, Uttar Pradesh, West Bengal; China, Indonesia, Iran, Laos, Malaysia, Myanmar, Nepal, Thailand, and Vietnam $[4,29,43]$.

\subsection{Tribe: Dorcaschematini}

- Olenecamptus bilobus (Fabricius, 1801) (Figure 2(b)). Saperda biloba Fabricius 1801, Bibl. Acad. Nov., 2: 324.

Material Examined. 1 ex., Rajabhatkhawa, BTR, 19.v.1995, coll. S. Saha; 1 ex., Nimati, BTR, 27.v.1997, coll. D. Raychaudhuri; 1 ex., Rajabhatkhawa, BTR, 29.iii.2002(LT), coll. S. Sarkar; 1 ex., Rajabhatkhawa, BTR, 11.x.2009, coll. S. Sarkar; 1 ex., Holapara, JWLS, 29.iv.2003, coll. S. Bhattacharya.

Distribution. India: Andaman and Nicobar Island, Maharashtra, Orissa, Tripura, Uttarakhand, West Bengal; Australia, Bhutan, Bismarck A., Celebes Island, China, Comores, Indonesia, Japan, Laos, Madagascar, Malaysia, Malacca, Mauritius, Micronesia, Myanmar, Nepal, New Guinea, Pakistan, Philippines, Reunion Island, Sunda Island, Seychelles Island, Sri Lanka, Taiwan, Thailand, Timor, Vanuatu Island, and Vietnam [4, 12, 22, 23, 26, 34, 37].

- Olenecamptus dominus Thomson, 1860 (Figure 2(c)).

Olenecamptus dominus Thomson, 1860, Essai class. Cer. 362.

Material Examined. 1 ex., Damanpur, BTR, 12.iv.2009, coll. S. Sarkar; 2 ex., Hollong, JWLS, 29.iv.2002, coll. S. Bhattacharya. 
Distribution. India: Assam, West Bengal; Cambodia, China, Laos, Thailand, Vietnam $[3,4,37]$.

\subsection{Tribe: Lamiini}

- Acalolepta griseipennis (Thomson, 1857) (Figure $2(\mathrm{~d})$ ).

Monochammus griseipennis Thomson, 1857, Arch. Ent. 1: 296.

Material Examined. 2 ex., Newland, BTR, 17.v.1997, coll. S. Saha; 1 ex., Nimati, BTR, 22.v.1997, coll. B. Kundu; 1 ex., Kunjanagar, JWLS, 27.iv.2002, coll. S. Bhattacharya; 1 ex., Bichabhanga, GNP, 5.vi.2006, coll. S. Sen.

Distribution. India: Sikkim, West Bengal; Bhutan, China, Laos, Malaysia, Myanmar, and Nepal [3, 4, 37, 39].

- Aristobia approximator (Thomson, 1865) (Figure 2(e)).

Celosterna approximator Thomson, 1865, Mem. Soc. R. Sci. Liege, 19: 552.

Material Examined. 1 ex., Gadadhar, BTR, 20.x.2003, coll. S. Sarkar; 1 ex., Cheko, BTR, 11.ix.2009, coll. S. Sarkar.

Distribution. India: Assam, Manipur, West Bengal; Cambodia, China, Laos, Malaysia, Myanmar, Thailand, and Vietnam $[3,4,12,37,39,44]$.

Blepephaeus succinator (Chevrolat, 1852) (Figure 2(f)).

Monohammus succinator Chevrolat, 1852, Rev. Mag. Zool. 2, 4: 417 .

Material Examined. 1 ex. Panbari, BTR, 25.v.2009, coll. S. Sarkar; 2 ex., Mendabari, JWLS, 3.vii.2003, coll. S. Sarkar; 1 ex., Gorumara, GNP, 3.vi.2006, coll. S. Sen.

Distribution. India: Assam, Meghalaya, Sikkim, West Bengal; China, Indonesia, Laos, Malacca, Malaysia, Nepal, Taiwan, Thailand, and Vietnam $[3,4,29,37]$.

Macrochenus isabellinus Aurivillius, 1920 (Figure 3(a)).

Macrochenus isabellinus Aurivillius, 1920, Ark. Zool., 13 (9): 372.

Material Examined. 1 ex., Phaskhawa, BTR, 25.v.1997, coll. S. Saha.

Distribution. India: West Bengal; China, Laos, Myanmar, Nepal, Thailand, and Vietnam $[4,38,39]$.

- Paraleprodera insidiosa (Pascoe, 1888) (Figure 3(b)).

Leprodera insidiosa Pascoe, 1888, Ann. Mag. Nat, Hist. ser. 6, 2: 391.

Material Examined. 1 ex., Panbari, BTR, 24.v.1995, coll. B. Kundu; 1 ex., Raimatang, BTR, 30.v.1996, coll. S. Saha; 1 ex.,

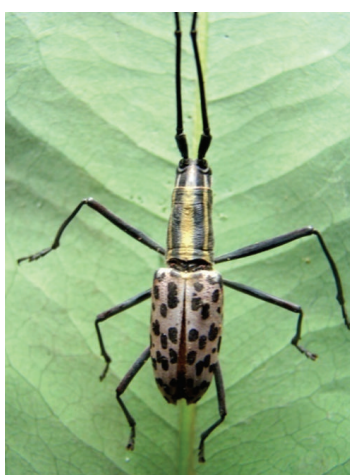

(a)

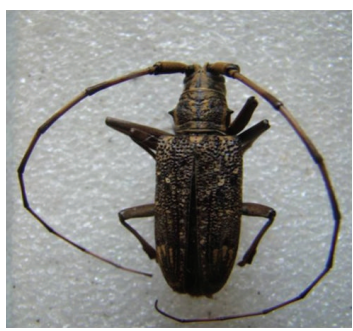

(c)

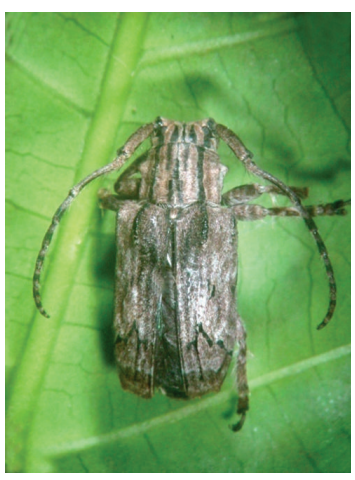

(e)

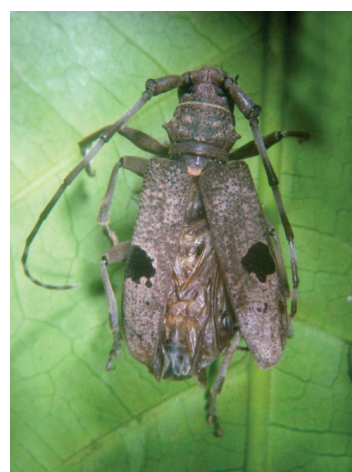

(b)

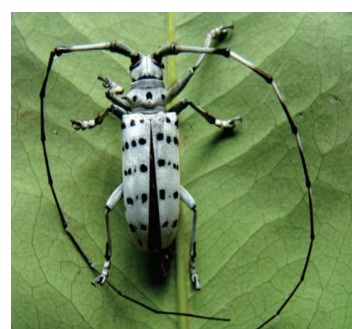

(d)

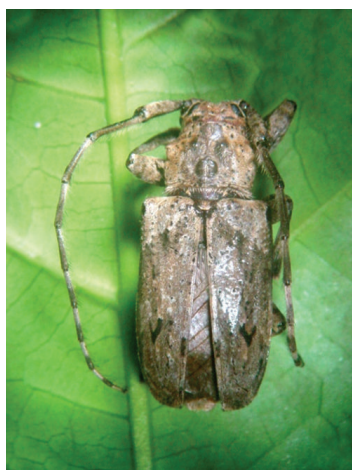

(f)
FIgURE 3: (a) Macrochenus isabellinus Aurivillius, (b) Paraleprodera insidiosa (Pascoe), (c) Pharsalia (Cycos) subgemmata (Thomson), (d) Pseudonemophas versteegi (Ritsema), (e) Aesopida malasiaca Thomson, and (e) Coptops aedificator (Fabricius).

Sankos, BTR, 10.vi.2003, coll. S. Sarkar; 1 ex., S. Bholka, BTR, 12.iv.2009, coll. S. Sarkar; 2 ex., Jayanti, BTR, 14.iv.2009, coll. S. Sarkar; 1 ex., Rajabhatkhawa, BTR, 24. v.2009, coll. S. Sarkar; 1 ex., Panbari, BTR, 25.v.2009, coll. S. Sarkar; 1 ex., Rajabhatkhawa, BTR, 12.iv.2010, coll. S. Sarkar; 1 ex., Malangi, JWLS, 23.iii.2002, coll. S. Bhattacharya; 1 ex., Chapramari, CWLS, 6.vi.2006, coll. S. Sen.

Distribution. India: North India, West Bengal; Cambodia, China, Laos, Malaysia, Myanmar, Taiwan, Thailand, and Vietnam $[3-5,39]$.

- Pharsalia (Cycos) subgemmata (Thomson, 1857) (Figure 3(c)).

Monochamus subgemmatus Thomson, 1857, Arch. Ent. 1: 294. 
Material Examined. 1 ex., Kumargram, BTR, 25.vi.2004, coll. S. Sarkar.

Distribution. India: Andaman and Nicobar Islands, West Bengal; Bangladesh, Cambodia, China, Indonesia, Laos, Malaysia, Myanmar, Nepal, Thailand, and Vietnam [3, 4, 22, 39].

- Pseudonemophas versteegi (Ritsema, 1881) (Figure $3(\mathrm{~d}))$.

Monohammus versteegi Ritsema, 1881. Not. Leyd. Mus., 3(3): 155.

Material Examined. 1 ex., Tashigaon, BTR, 26.v.1996, coll. B. Kundu; 2 ex., Newland, BTR, 17.v.1997, coll. D. Raychaudhuri; 1 ex., Jayanti, BTR, 21.iv.2003, coll. S. Saha; 1 ex., Hatipota, BTR, 15.iv.2009, coll. S. Sarkar; 1 ex., Raimatang, BTR, 27.v.2009, coll. S. Sarkar; 1 ex., Dima BTR, 10.iv.2010, coll. S. Sarkar; 1 ex., Gorumara, GNP, 5.vi.2004, coll. M. K. Biswas; 2 ex., Gorumara, GNP, 5.vi.2004, coll. M. K. Biswas.

Distribution. India: Sikkim, West Bengal; China, Indonesia, Laos, Malaysia, Myanmar, Nepal, Thailand, and Vietnam [3, $4,37,39]$.

\subsection{Tribe: Mesosini}

Aesopida malasiaca Thomson, 1864 (Figure 3(e)).

Aesopida malasiaca Thomson, 1864, Mem. Soc. R. Sci. Liege 19: 62.

Material Examined. 1 ex., Chapramari, CWLS, 4.iv.2003, coll. M. K. Biswas.

Distribution. India: West Bengal; China, Indonesia, Laos, Malaysia, and Vietnam [3, 4, 39].

- Coptops aedificator (Fabricius, 1792) (Figure 3(f)).

Lamia aedificator Fabricius, 1792, Hafniae, Proft, 1, 2: 275.

Material Examined. 1 ex., Kumargram, BTR, 14.iv.2003, coll. S. Sarkar.

Distribution. India: Andaman Island, West Bengal; Cameroon, Cape Verde, Central African Republic, China, Comoros, Democratic Republic of Congo, Djibouti, Ethiopia, Fernando Po, Gabon, Hawaii, Ivory coast, Mauritius, Reunion Island, Kenya, Liberia, Madagascar, Malawi, Mauritius, Namibia, Nigeria, Philippines, Principe Island, Saudi Arabia, Senegal, Seychelles, Somalia, South Africa, Sri Lanka, Taiwan, Tanzania, Uganda, Yemen, Zambia [3-5, 39, 42].

Coptops leucostictica White, 1858 (Figure 4(a)).

Coptops leucostictica White, 1858, Ann. Mag. Nat. Hist., 3, $2: 272$.

Material Examined. 1 ex., Rajabhatkhawa, BTR, 7.iii.2003, coll. S. Sarkar; 1 ex., Sankos, BTR, 26.v.2004, coll. S. Sarkar; 1 ex., Rajabhatkhawa, BTR, 24.v.2009, coll. S. Sarkar; 1 ex.,

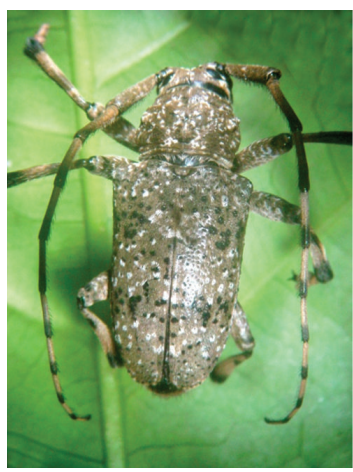

(a)

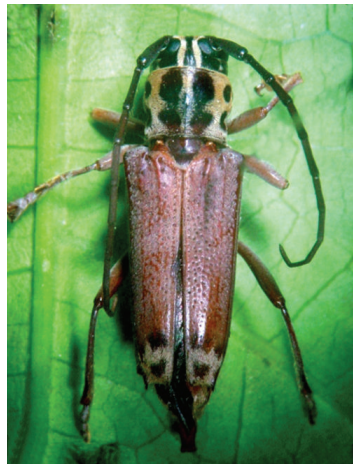

(c)

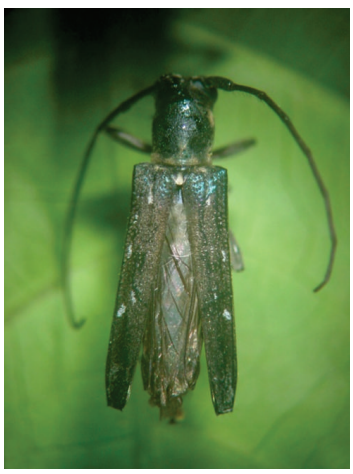

(e)

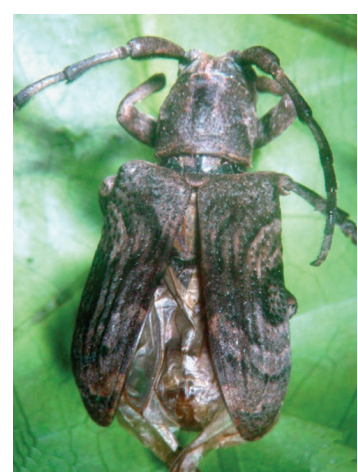

(b)

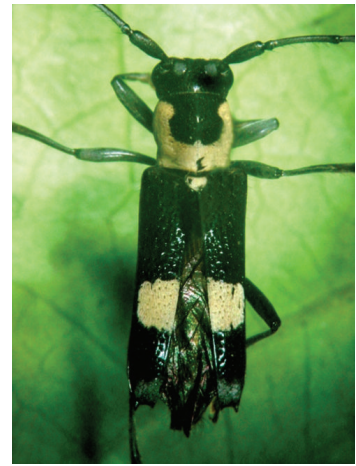

(d)

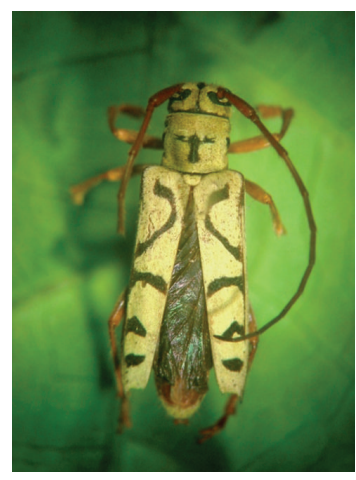

(f)
Figure 4: (a) Coptops leucostictica White, (b) Pterolophia (Hylobrotus) lateralis Gahan, (c) Glenea (Stiroglenea) cantor (Fabricius), (d) Glenea (s str.) indiana (Thomson), (e) Glenea (s. str.) pulchra Aurivillius, and (f) Glenea (s. str.) t-notata Gahan.

Rajabhatkhawa, BTR, 13.x.2009, coll. S. Sarkar; 1 ex., Madarihat, JWLS, 7.6.2002, coll. S. Bhattacharya.

Distribution. India: Assam, Sikkim, West Bengal; Cambodia, China, Indonesia, Laos, Malaysia, Myanmar, Thailand, and Vietnam $[4,5,29]$.

\subsection{Tribe: Pteropliini}

Pterolophia (Hylobrotus) lateralis Gahan, 1894 (Figure $4(\mathrm{~b}))$.

Pterolophia lateralis Gahan, 1894, Ann. Mus. Civ. Genova., $34: 65$. 
Material Examined. 1 ex., Madarihat, JWLS, 7.6.2002, coll. S. Bhattacharya.

Distribution. India: West Bengal; China, Indonesia, Japan, Laos, Myanmar, Taiwan [3-5, 37, 39].

\subsection{Tribe: Saperdini}

- Glenea (Stiroglenea) cantor (Fabricius, 1787) (Figure $4(\mathrm{c}))$.

Lamia cantor Fabricius, 1787, Mant. Ins. 1: 142.

Material Examined. 1 ex., Jayanti, BTR, 24.v.1996, coll. S. Saha; 1 ex., Kumargram, BTR, 18.v.1997, coll. D. Raychaudhuri; 1 ex., Garam, BTR, 20.vi.2003, coll. S. Sarkar; 2 ex., Bendaki, JWLS, 8.xi.2001, coll. S. Bhattacharya; 2 ex., Daidaighat, Jaldapara, 5.3.2003, coll. S. Bhattacharya; 1 ex., Jaldapara, JWLS, 7.xi.2004, coll. S. Bhattacharya; 1 ex., Gorumara, GNP, 2.xi.2003, coll. M. K. Biswas.

Distribution. India: West Bengal; China, Laos, Philippines, and Vietnam $[3,4]$.

Glenea (s. str.) indiana (Thomson, 1857) (Figure 4(d)).

Stibara indiana Thomson, 1857, Archive Ent., 1: 141.

Material Examined. 1 ex., Rajabhatkhawa, BTR, 19.v.1995, coll. B. Kundu; 1 ex., Panbari, BTR, 20.v.1995, coll. D. Raychaudhuri; 1 ex., Newland, BTR, 17.v.1997, coll. S. Saha; 1 ex., Gadadhar, BTR, 5.ix.2009, coll. S. Sarkar.

Distribution. India: Meghalaya, Sikkim, Tripura, West Bengal; Bangladesh, Bhutan, Indonesia, Malaysia, Myanmar, Nepal, Laos, Thailand, and Vietnam [3, 4, 12, 26, 29, 37, 40, 45].

Glenea (s. str.) pulchra Aurivillius, 1926 (Figure 4(e)).

Glenea pulchra Aurivillius, 1926, Philip. J. Sci. 30: 111.

Material Examined. 1 ex., S. Bholka, BTR, 20.iii.1997, coll. S. Saha; 1 ex., Kunjanagar, JWLS, 3.vi.2003, coll. M. K. Biswas; 1 ex., Siltorsa, JWLS, 19.x.2003, coll. S. Bhattacharya; 1 ex., Budhuram, GNP, 4.vi.2006, coll. M. K. Biswas.

Distribution. India: Assam, Sikkim, West Bengal; China, Indonesia, Laos, Malacca, Malaysia, Myanmar, Philippines, Taiwan, Thailand, and Vietnam [3, 4, 12, 37, 39].

- Glenea (s. str.) t-notata Gahan, 1889 (Figure 4(f)).

Glenea (s. str.) t-notata Gahan, 1889, Trans. Ent. Soc., 223.

Material Examined. 1 ex., Newland, BTR, 17.vii.1997, coll. D. Raychaudhuri.

Distribution. India: West Bengal; Bangladesh, China, Laos, Nepal [3-5, 37, 39].

Nupserha fricator (Dalman, 1817) (Figure 5(a)).

Saperda fricator Dalman, 1817, Syn. Ins. 1 (3): 183.

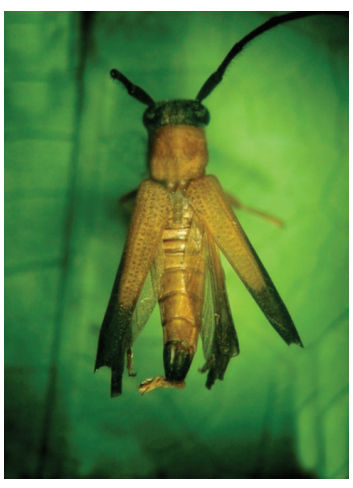

(a)

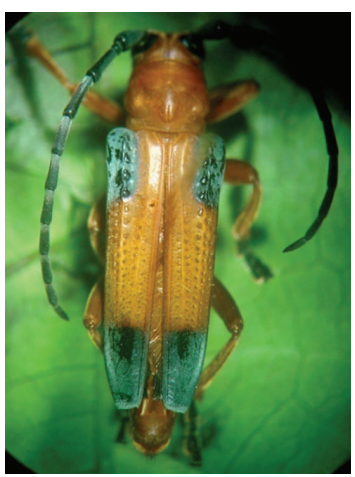

(c)

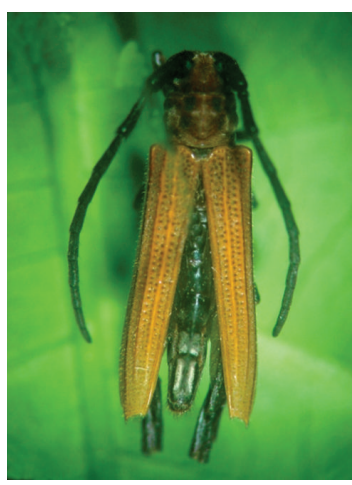

(b)

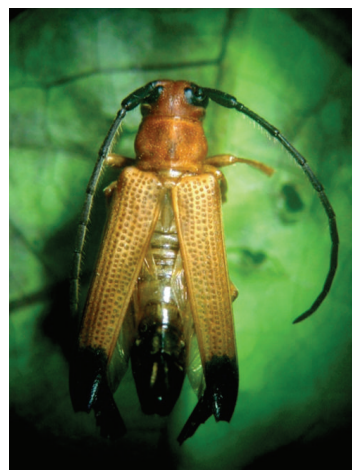

(d)

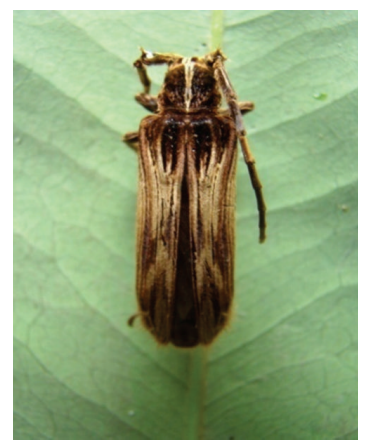

(e)

FIGURE 5: (a) Nupserha fricator (Dalman), (b) Nupserha quadrioculata (Thunberg) (c) Stibara (s. str.) tetraspilota Hope, (d) Stibara (s. str.) tricolor (Fabricius), and (e) Xylorhiza adusta (Wiedmann).

Material Examined. 1 ex., Newland, BTR, 19.v.1997, coll. D. Raychaudhuri; 1 ex., Bichabhanga, GNP, 1.iv.2003, coll. M. K. Biswas.

Distribution. India: Arunachal Pradesh, Sikkim, West Bengal; Celebes Island, China, Indonesia, Malaysia, Myanmar, Nepal, Philippines, Taiwan, Thailand, and Vietnam $[3,4,29,46]$.

Nupserha quadrioculata (Thunberg, 1787) (Figure $5(\mathrm{~b}))$.

Saperda quadrioculata Thunberg, 1787, Mus. Nat. Acad. Upsala 4: 57. 
Material Examined. 1 ex., Nimati, BTR, 3.iv.2002, coll. S. Sarkar; 1 ex., S. Bholka, BTR, 12.iv.2009, coll. S. Sarkar.

Distribution. India: West Bengal; China, Indonesia, Laos, Myanmar, Nepal, Thailand, and Vietnam [3, 4, 37, 39].

Stibara (s. str.) tetraspilota Hope, 1840 (Figure 5(c)).

Stibara tetraspilota Hope, 1840, Proc. Zool. Soc. Lond., 1: 79 .

Material Examined. 1 ex, Phaskhawa, BTR, 9.v.1994, coll. B. Kundu; 1 ex, Panbari, BTR, 20.v.1995, coll. S. Saha; 1 ex, Phaskhawa, BTR, 25.v.1996, coll. S. Saha; 1 ex, S. Bholka, BTR, 19.vi.1996, coll. S. Saha; 1 ex, S. Bholka, BTR, 19.v.1997, coll. D. Raychaudhuri; 1 ex, Nimati, BTR, 21. v.1997, coll. S. Saha; 1 ex, Nimati, BTR, 3.iv.2002, coll. S. Sarkar; 1 ex, Kumargram, BTR, 11.iv.2009, coll. S. Sarkar; 1 ex, Jaldapara, JWLS, 29.iv.2002, coll. S. Bhattacharya; 1 ex, Hasimara, JWLS, 10.vi.2002, coll. S. Bhattacharya; 1 ex, Baradabri, JWLS, 6.vi.2002 coll. S. Bhattacharya; 1 ex, Chapramari, CWLS, 6.vi.2006, coll. S. Sarkar.

Distribution. India: Assam, Himachal Pradesh, Sikkim, Meghalaya, Orissa, West Bengal; Laos, Myanmar, Thailand $[4,23,25,29,33,37]$.

- Stibara (s. str.) tricolor (Fabricius, 1792) (Figure $5(\mathrm{~d}))$.

Saperda tricolor Fabricius, 1792, Hafniae, Proft., 1 (2): 309.

Material Examined. 2 ex., Newland, BTR, 26.v.2004, coll. S. Sarkar.

Distribution. India: Assam, West Bengal; China, Laos, Malaysia, Myanmar, Thailand, and Vietnam [3-5, 37].

\subsection{Tribe: Xylorhizini}

Xylorhiza adusta (Wiedmann, 1819) (Figure 5(e)).

Lamia adusta Wiedmann, 1819, Zool. Mag. Kiel., 1 (3): 182.

Material Examined. 1 ex., TEC, JWLS, 9.vi.2003, coll. S. Bhattacharya.

Distribution. India: Orissa, Sikkim, Tamil Nadu, West Bengal; Cambodia, China, Indonesia, Laos, Malacca, Malaysia, Myanmar, Nepal, Pakistan, Philippines, Sri Lanka, Taiwan, Thailand, and Vietnam [3-5, 23, 29, 37, 39].

\section{Acknowledgments}

The authors sincerely acknowledge the financial assistance rendered by Ministry of Environment \& Forests, Government of India (Sanction no. 4206/WZ/2W-231/94 dt. 01.11.1994 \& 14/34/2000-ERS/RE dt. 17.10.2001), National Tea Research Foundation, C/o Tea Board, Government of India (Sanction no. RL: 17(9)/2000/808 dt. 4/8.10.2001), West Bengal
Biodiversity Board, Government of West Bengal (Sanction no. 326/5k(Bio)-3/2007 dt. 11.12.2008 and 21/5k(Bio)$3 / 2007$ dt. 14.01.2009) and University Grants Commission, Eastern Regional Office (Sanction no. FPSW-036/08-09 dt. 5.12.2008); Director, Zoological Survey of India, Directorate of Forests, Government of West Bengal and Head, Department of Zoology, University of Calcutta for necessary support.

\section{References}

[1] H. Özdikmen and U. Çaglar, "Contribution to the knowledge of longhorned beetles (Coleoptera, Cerambycidae) from Turkey. Subfamilies: Prioninae, Lepturinae, Spondylidinae and Cerambycinae," Journal of the Entomological Research Society, vol. 6, no. 1, pp. 39-69, 2004.

[2] S. Ponpinij, P. Hormchan, and V. Rojanavongse, "Checklist of new records of subfamily Lamiinae (Coleoptera: Cerambycidae) in northern Thailand," Kasetsart Journal, vol. 45, no. 5, pp. 841-855, 2011.

[3] J. Roguet, "Lamiaires du monde," June 2012, http://www.lamiinae.org/73v/.

[4] F. Bisby, "Catalogue of life," June 2012, http://www.catalogueoflife.org/annual-checklist/.

[5] F. Vitali, Cerambycoidea.com, June 2012, http://www.cerambycoidea.com/.

[6] E. P. Stebbing, Indian Forest Insects of Economic Importance: Coleoptera, Eyre and Spottiswoode, London, UK, 1914.

[7] C. Aurivillius, "Coleopterorum catalogus," in Cerambycidae: Lamiinae II, W. Junk and S. Schenkling, Eds., vol. 23 of Pars 74, pp. 323-704, Berlin, Germany, 1923.

[8] W. S. Fisher, "New Cerambycidae from India," Indian Forest Records, vol. 17, no. 6, pp. 1-8, 1933.

[9] W. S. Fisher, "New Cerambycidae from India, II (Coleoptera)," Indian Forest Records, vol. 6, no. 5, pp. 197-202, 1940.

[10] C. F. C. Beeson and B. M. Bhatia, "On the biology of the Cerambycidae (Coleoptera)," Indian Forest Records, vol. 5, no. 1, pp. 1235, 1939.

[11] G. D. Bhasin and M. L. Roonwal, "A list of insect pests of forest plants in India and the adjacent countries," Indian Forest Bulletin, vol. 17, no. 1, pp. 93-97, 1954.

[12] C. F. C. Beeson, The Ecology and Control of Forest Insects of India and the Adjoining Countries, Government of India, 1961.

[13] S. Breuning, "Revision systematique des especes du genre Oberia mulsant du globe," Frustula Entomologica, part 1, 2, 3, pp. $1-232,1960$.

[14] S. Breuning, "Bestimmungstabella der Lamiiden-Triben nebst revision der pteroplini der asiatischen region (Col. Ceramb.) 1," Entomologischen Arbeiten aus dem Museum G. Frey, vol. 13, no. 2, pp. 371-493, 1962.

[15] S. Breuning, "Bestimmungstabelle der Lamiiden-Triben nebst revision der pteroplini der asiatischen region (Col. Ceramb.) 111," Entomologischen Arbeiten aus dem Museum G. Frey, vol. 14, pp. 168-251, 1963.

[16] S. Breuning, "Bestimmungstabelle der Lamiiden-Triben nebst revision der pteroplini der asiatischen region (Col. Ceramb.) 111," Entomologischen Arbeiten aus dem Museum G. Frey, vol. 14, pp. 466-537, 1963.

[17] S. Breuning, "Revision der apomecynini der asiatisch-australischen region," Entomologische Abhandlungen Museum für Tierkunde in Dresden, vol. 30, pp. 1-528, 1964. 
[18] S. Breuning, "Revision der 35 gattung der pteroplini der asiatischen region (Col. Ceramb.)," Entomologischen Arbeiten aus dem Museum G. Frey, vol. 16, pp. 161-472, 1965.

[19] S. Breuning, "Revision der agapanthini der eurasiatisch-australischen region (Col. Ceramb.)," Entomologische Abhandlungen Museum für Tierkunde in Dresden, vol. 34, no. 1, pp. 1-144, 1966.

[20] T. N. Khan and P. K. Maiti, "The bionomics of the round-head borer, Olenecamptus bilobus (Fabricius) (Coleoptera: Cerambycidae)," Proceedings of the Zoological Society, vol. 33, no. 1-2, pp. 71-85, 1980.

[21] T. N. Khan and P. K. Maiti, "Studies on the biotaxonomy, biology and ecology of some longicorn beetle borers (Coleoptera: Cerambycidae) of the Islands of Andaman, India," Records of the Zoological Survey of India, no. 45, pp. 1-100, 1983.

[22] T. N. Khan, "A biotaxonomic key to the Cerambycidae (Coleoptera) of Andaman and Nicobar Islands," Journal of Bengal Natural History Society B, vol. 8, no. 2, pp. 14-29, 1989.

[23] S. Biswas and P. K. Basak, "Insecta: Coleoptera: Cerambycidae," Zoological Survey of India: State Fauna Series, pp. 185-195, 1993.

[24] L. K. Jha and P. K. Sensarma, Forest Entomology, Ashish Publishing House, New Delhi, India, 1994.

[25] P. Mukhopadhyay and S. Biswas, "Coleoptera: Cerambycidae," Zoological Survey of India: State Fauna Series, pp. 41-67, 2000.

[26] P. Mukhopadhyay and S. Biswas, "Coleoptera: Cerambycidae," Zoological Survey of India: State Fauna Series, pp. 139-142, 2000.

[27] S. Saha and D. Raychaudhuri, "Lamiids (Cerambycidae) of Buxa Tiger Reserve, Jalpaiguri, West Bengal," Insect Environment, vol. 5, no. 4, article 185, 2000.

[28] T. Dalui, C. Ray, S. Saha, and D. Raychaudhuri, "Studies on the borers attacking shade trees of tea plantations," in Proceedings of the 3rd NTRF Workshop: Tea Research-New Trend, pp. 55-56, Kolkata, India, April 2002.

[29] P. Mukhopadhyay and S. K. Halder, "Insecta: Coleoptera: Cerambycidae," Zoological Survey of India: State Fauna Series, pp. 181-199, 2003.

[30] G. Mathew, R. S. M. Shamsudeen, R. Chandran, and C. M. Brijesh, "Fauna of protected areas-23: insect fauna of Peppara Wildlife Sanctuary, Kerala, India," Zoos' Print Journal, vol. 19, no. 11, pp. 1680-1683, 2004.

[31] G. Mathew, R. S. M. Shamsudeen, and R. Chandran, "Fauna of protected areas-15: insect fauna of Peechi-Vazhani Wildlife Sanctuary, Kerala, India," Zoos' Print Journal, vol. 20, no. 8, pp. 1955-1960, 2005.

[32] A. Sen and H. V. Ghate, "A recent record of Astathes bimaculata (Fabricius) (Coleoptera: Cerambycidae: Lamiinae) from Kerala," Zoos' Print Journal, vol. 20, no. 8, article 1965, 2005.

[33] A. Sen and H. V. Ghate, "A short note on three species of Stibara (Cerambycidae: Lamiinae: Saperdini)," Zoos' Print Journal, vol. 21, no. 3, article 2198, 2006.

[34] H. V. Ghate and A. Sen, "A note on three species of the genus Olenecamptus chevrolat (Coleoptera: Cerambycidae: Lamiinae) found in India," Zoos' Print Journal, vol. 21, no. 12, pp. 2514-2515, 2006.

[35] O. T. Singh, J. Chakravorty, and R. Varatharajan, "Entomofauna of Kane Wildlife Sanctuary, Arunachal Pradesh, north eastern India," Journal of Threatened Taxa, vol. 2, no. 13, pp. 1392-1400, 2010.

[36] J. K. Jonathan and P. P. Kulkarni, Manual, Collection, Preservation and Identification of Insects and Mites of Economic Importance, Edited by B. K. Tikader, Zoological Survey of India, 1986.
[37] J. A. Rondon and S. von Breuning, "Lamiines du laos," in Cerambycid-Beetles of Laos (Longicornes du Laos), J. L. Gressitt, J. A. Rondon, and S. von Breuning, Eds., vol. 24 of Pacific Insects' Monograph, pp. 315-571, Bishop Museum, Honolulu, Hawaii, USA, 1970.

[38] V. K. Thapa, An Inventory of Nepal's Insect: Volume III, (Hemiptera, Hymenoptera, Coleoptera \& Diptera), IUCN-The World Conservation Union, Lalitpur, Nepal, 2000.

[39] “Global species," June 2012, http://www.globalspecies.org/.

[40] M. Hayashi, "On some longicorn beetles of Nepal (Coleoptera: Cerambycidae)," Bulletin of the Osaka Jonan Women's Junior College, vol. 14, pp. 1-20, 1981.

[41] S. Ponpinij, P. Hormchan, and V. Rojanavongse, "Checklist of new records of subfamily Lamiinae (Coleoptera: Cerambycidae) in northern Thailand," Kasetsart Journal, vol. 45, no. 5, pp. 841-855, 2011.

[42] D. J. Heffern, "Catalog and bibliography of longhorned beetles from the HawaiianIslands (Coleoptera: Cerambycidae)," October 2011, http://www.docstoc.com/docs/18543809/Catalogand-Bibliography-of-Longhorned-Beetles-from-the-Hawaiian.

[43] H. Özdikmen, H. Ghahari, and S. Turgut, "New records for Palaearctic Cerambycidae from Iran with zoogeographical remarks (Col.: Cerambycoidea: Cerambycidae)," Munis Entomology \& Zoology Journal, vol. 4, no. 1, pp. 1-14, 2009.

[44] P. Mukhopadhyay and S. K. Halder, "Insecta: Coleoptera: Cerambycidae," Zoological Survey of India: State Fauna Series, pp. 421-431, 2004.

[45] M. Lin, O. Montreuil, G. Tavakilian, and X. Yang, "Reinstatement of the genus Heteroglenea Gahan, with four new combinations, four new synonyms and three new species (Coleoptera: Cerambycidae: Lamiinae: Saperdini)," Zootaxa, vol. 2137, pp. 122, 2009.

[46] H. Makihara, A. N. Woro, and Sugiarto, "Longicorn beetles from Gunung Halimun National Park, West Java, Indonesia from 1997-2002 (Coleoptera, Disteniidae and Cerambycidae)," Bulletin of FFPRI, vol. 1, no. 3, pp. 189-223, 2002. 

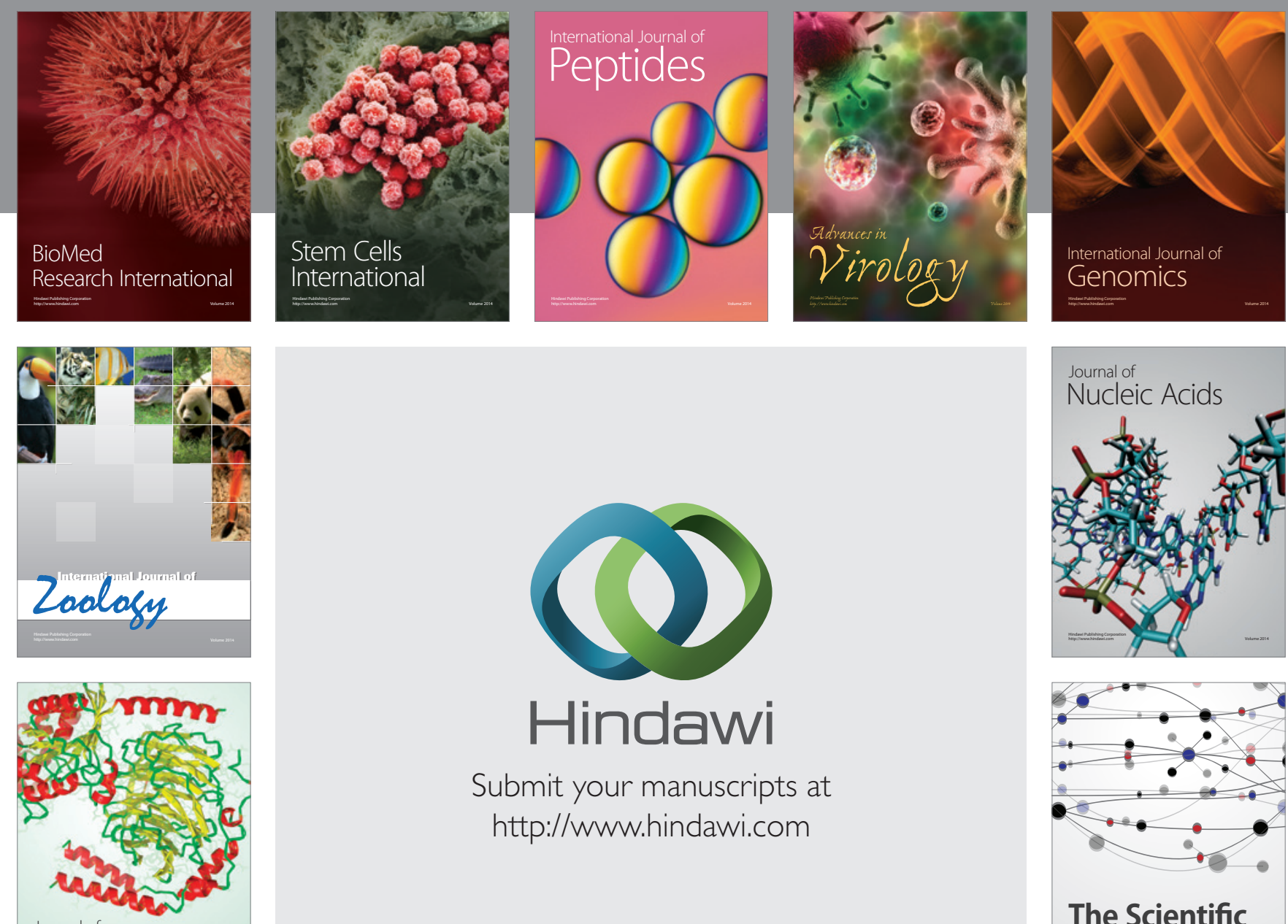

Submit your manuscripts at

http://www.hindawi.com

Journal of
Signal Transduction
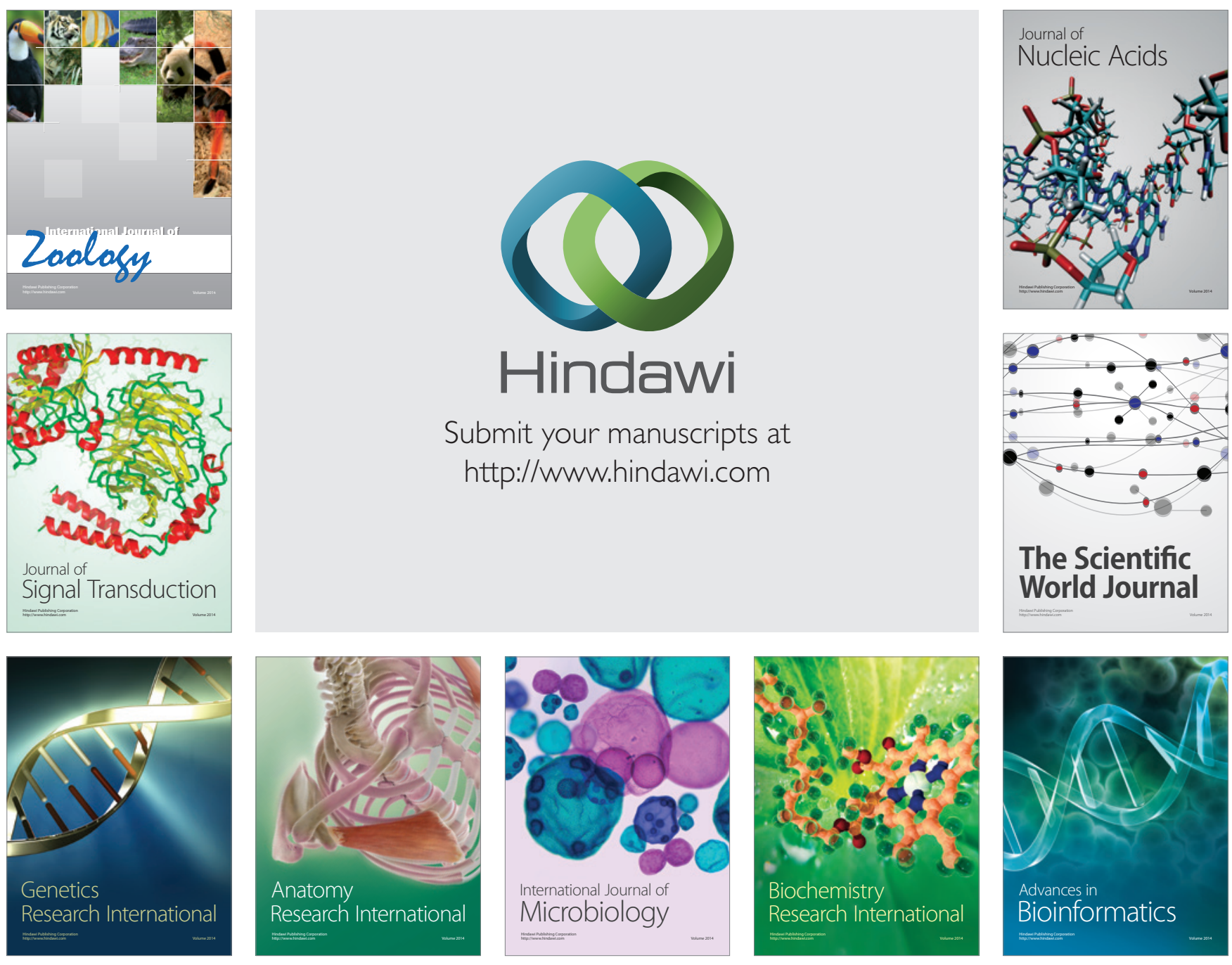

The Scientific World Journal
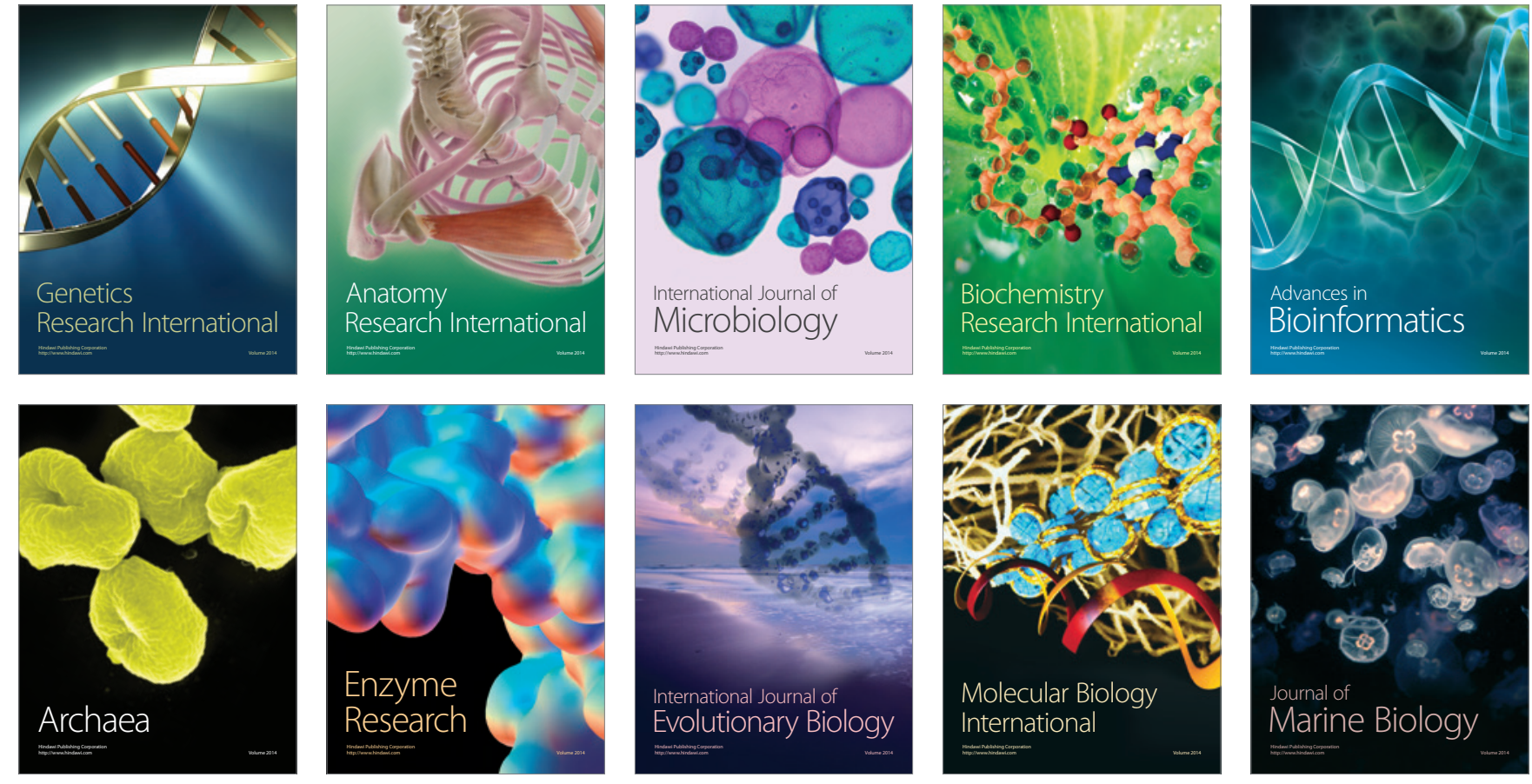